\title{
Implementation Technologies and Procedure of the Real-time Interactive Roaming System of Virtual City
}

\author{
Pu Liu ${ }^{*}$ and Zhenghong Peng
}

School of Urban Design, Wuhan University, Wuhan, Hubei430072, China

\begin{abstract}
Virtual reality is a hot research topic in computer science and the introduction of virtual roaming to the field of urban planning provides a new means for urban design. This paper analyzes the development, features and technology of virtual reality and the virtual city roaming and its applications and advantages in urban planning; then focus on key technologies and how to implement a real-time interactive virtual city roaming system, taking example of the scene development software Vega, and finally concludes the importance of virtual city roaming in urban planning.
\end{abstract}

Keywords: Vega, virtual reality, virtual roaming, virtual city, computer emulation, digital city, urban planning, visualization.

\section{INTRODUCTION}

A virtual city is a concept proposed after digital city. A digital city is digitization, networking and visualization of urban geography, environment, resources, population, economy and various social services in order to optimize decision support. While a virtual city is a manifestation used for description and a virtual reproduction, the operating environment and interactive interface of a digital city and a means of true three-dimensional display of city information. From the base layer to the application layer and to the decision-making layer, a virtual city participates in each layer of a digital city.

The virtual city roaming replaces traditional maps to explain, describe, analyze and discuss design ideas and urban progress, presents a city vividly in front of people, provides an immersive environment for urban development and the application of geographic information and enables users to move and interact in it. It enables users to visually see the urban street scope, to autonomously roam in the threedimensional scene and to undertake operations such as query, measurement and flight browsing, so as to understand the direction of future planning and development of enterprises, building districts and even cities as well as to provide visualized geospatial information services for urban construction planning, community services, property management, tourism, transportation, fire safety and so on.

This paper introduces a virtual city roaming system, makes comparative analysis of other computer-aided methods commonly used in urban planning and design, researches the composition, key technologies and realizing methods of the virtual city roaming and makes a detailed description of the process and technologic route to achieve the virtual city roaming system with MutligenVega. Finally, it makes a brief summary of the application and significance of virtual reality roaming technology in the field of urban construction.

*Address correspondence to this author at the School of Urban Design, Wuhan University, Wuhan, Hubei 430072, China; Tel: (+86)13871231870; Fax: (+86-027)68773062; E-mail: liu2005xin@163.com

\section{VIRTUAL REALITY AND VIRTUAL CITIES}

Virtual reality provides users with the simulated operating environment to make users feel like being in the real world and form a realistic visual, auditory and tactile sensory world and also enables to implement interactive feedback. The term of virtual reality was first proposed in the 1980s by an American scientist named Jaron Lanier. In 1993, the text of "Virtual Reality System and Application" published by BurdeaG and Philippe Coiffet proposed the basic characteristics of virtual reality, namely, the "triangle" of triune "Immersion-Interaction-Imagination".

The virtual city is the application of virtual reality technology in the field of urban planning and architecture. Its origin can be traced back to the early 1980s with the 3D simulation of two cities of Skidmore and Merrill in the United States. After the 1990s, virtual city technology was gradually extended from producing urban models and urban maps to urban construction and urban planning and to designs of large-scale public construction projects and urban environment.

Virtual city is able to transfer abstract data in geographic information system and other database into the intuitive and perceptible space environment to facilitate observation and to generate auxiliary performance integrated systems, such as the landscape performance system and the transportation planning system. Urban planners can build a wealth of information of a city such as roads, buildings, residential areas and commercial outlets into database and transform into the virtual environment; and then enter the virtual environment through the man-machine dialogue tools and get to know and judge the advantages and disadvantages of various planning programs with different dominant factors through personal observation and experience. With the virtual city, the effect of the implementation of a program can be pre-tested without any real implementation of the planning scheme to see if it can achieve the comprehensive benefits in economic, social and ecological aspects and it can repeatedly modify, improve and assist the formulation of the final decision- 
making program [1]. Therefore, it can not only improve the scientific nature of urban planning or urban ecological construction, reduce the cost of urban development, but also reduce the time for planning and design. It is also available for the general public to show the design results and project presentation and demonstration is implemented in the virtual three-dimensional environment where users make immersive and all-round review in the dynamic and interactive way and can not only be immersed in the virtual environment, but also undertake query, analysis, evaluation, planning and decision-making.

The virtual urban technology includes a comprehensive variety of techniques including: virtual environment modeling, real-time display of complex scenes, mechanical feedback, tactile feedback, human-computer interaction, stereo synthesis, speech recognition and input, and system integration technology.

\section{VIRTUAL CITY ROAMING AND ITS ACHIEVING}

\subsection{Virtual City Roaming}

Virtual roaming is an important branch of virtual reality technology. Based on real space or virtual space, the virtual city roaming system provides the real-time simulation of the virtual environment with realistic visual, auditory and tactile integration; with the necessary equipment, users can roam in it in a natural way, observe virtual objects from any angle, generate a sense like being in the situation in person and also operate objects in it and get feedback at the same time. Virtual city roaming system is of great application value for the field of urban planning and architectural design. The main objects studied in urban design are the structure of a city and various elements, including a city's buildings, streets, squares, water features and greenery, edge and entrance, sight corridor, skyline, commanding heights, etc. Therefore, in the design process, it needs a large number of spatial thinking in images and at the same time it needs to take urban users' feelings as the core to analyze the urban design elements and the relationship among the elements. Therefore, it needs a variety of techniques to assist thinking in images and spatial modeling.

\subsection{Shortcomings of Computer-Aided Design Commonly Used in Urban Planning}

The most common way used in computer-aided urban planning and design is to make use of effect pictures and $3 \mathrm{D}$ animation to present three-dimensional space of a city [2]. As city models need to be reduced in a large scale, users can only get a bird's-view image of a city. So the city's architectural space cannot be felt from the perspective of a normal person and the real feelings of urban people cannot be obtained. The effect pictures can only provide partial static visual experience. Although animation has strong expression in dynamic 3D, but it does not have a real-time interactivity; besides, to modify programs, to change the layout of building models or to change observation routes need to be recalculated, which is time-consuming and cannot largely aid designers to dynamically scrutinize and adjust programs. Therefore, designers must also work by depending on their own space imagination and design principles. In fact, effect pictures and animation can only be used for simple and fixed demonstration and reporting programs and cannot meet the needs of urban design.

\subsection{Advantages of Virtual city Roaming Application in Urban Planning}

Virtual city roaming system can be a good solution to the above problems. It not only changes the graphic design into $3 \mathrm{D}$ urban scenes and has a real-time interactivity. The sense of immersion and interactivity not only allows users to get the immersive visual experience, but also allows users to get real-time data of scenes [3]; besides, the video can be derived according to the user's roaming route. Users can customize various roaming simulating options and have a comprehensive look at the future buildings or urban regions in dynamic and interactive way in the virtual three-dimensional environment: observe the scene from any angle, any distance and any sophistication; free to choose and switch motion mode such as: walking, driving, flying, etc.; freely control the route; also achieve the real-time switching comparison of a variety of design options and environmental effects. Virtual scenes change with changes of the location of viewers in the scene; it can also modify, add and delete scene models in real time and support the scene layout program adjustments and information inquiry. Those are unique compared with other roaming technologies along a fixed route. Virtual city roaming system not only has advantages compared to conventional planar design drawings, but also is far better than the effect pictures and three-dimensional animation and as a VR application; it will become an important assisting design tool following CAD for planners and architects.

\subsection{Hardware and Software to Achieve Virtual City Roaming System}

Virtual city roaming system consists of software and hardware systems. The software system includes modeling software and presentation software. Modeling software commonly adopts VRML, MultiGenCreator and so on. Presentation software organizes the already created 3D terrain and objects in a scene, sets the scene's time, weather and light and movement modes of objects, sets manipulation modes of the controlled objects and the observer as well as the basic movement mode and completes the threedimensional model's application programming and interface. Hardware system includes a computer platform, virtual peripherals and a display system. Computer platform adopts workstation or server platform; virtual peripherals include location tracking, virtual sound, data gloves and interactive equipment. The display system includes head mounted display, tabletop metaphor, large perspective projection equipment and stereo glasses. Urban simulation can be presented in large virtual reality system such as PowerWall and also be browsed in general computer monitors or Internet.

\subsection{Four Approaches to Virtual City Roaming}

Roaming technology to build virtual scene based on geometric models. First, construct geometric elements in a virtual scene. OpenGL or Direct3D are insufficient to directly complete the huge roaming scene, so 3Ds Max, Maya, and Softimage are often used for modeling, and then export models through a plug that can be driven by programming language, and then set the lighting and texture mapping, and 
finally set the control parameters to achieve interactive purpose, collision detection of objects, route roaming or autonomous roaming. The inadequacies of this way are huge time consumption of making complex scene models and a very large amount of computing of rendering so it is easy to get stuck and cause system halt in the roaming process [4]. But if reducing the number of point, line, and surface is reduced in pursuit of smooth roaming, the effect of verisimilitude will be sacrificed.

Roaming technology to build a virtual scene based on image rendering technology. Take the pictures of the realworld scenes with a camera at a certain angle and azimuth, surround to be continuous images and then use image processing software to undertake stretching, compression, rotation, perspective transformation, compositing, cuts, etc., so as to stitch or sew into a 360 degree panoramic image and then use the space editor or programming to build a virtual three-dimensional roaming scene [5]. This technique synthesize scenes through real photos [6]; roaming effects and the program computing time has nothing to do with the complexity of a scene, thus greatly reducing the processing load of a computer. However, the roaming also has many disadvantages. First, it requires high-precision professional cameras and also has high requirement for the shooting angle, or the generated scenes may be easily distorted and it may affect seamless stitching; besides, shooting light, brightness and shadows should be as consistent as possible, otherwise, after stitching, color may be inconsistent. Secondly, since they are all photos, it cannot use special effects, pick up objects in real time, interact in the roaming process or undertake collision detection. Finally, as photos of some scenes cannot be taken in certain locations or those do not exist in reality or haven't yet completed, the technique has limitations and cannot be used to design and preview in advance.

Mixed roaming technology to build a virtual scene based on the geometric modeling and image rendering. Mix the above two techniques and learn from each other; the basic idea is to take advantage of the image method to construct the environment of a virtual scene; at the same time, use modeling method to undertake entity construction for objects interacting with users; it also ensures the realistic, real-time, interactive and immersive sense. The mixing method of simultaneous and actual as well as seamless connectivity has also brought a lot of technical difficulties. For example, the coordinate position and orientation of the entity model object and the object in the two-dimensional should be exactly matched in the coordinate system; the brightness, the shadow intensity and direction of the geometric model in the virtual light source should also be exactly matched with the two-dimensional image; with the change of the user's perspective and the change of the position, it should immediately generate a new image and as for the operation of the entity object, it's able to get immediate feedback and meet the real-time interactivity.

Roaming technology to build virtual scene based on digital photogrammetry. The representative threedimensional visual simulation software of this modeling method contains full digital photogrammetry system VirtuoZo, three-dimensional visual geographic information system IMAGIS, city modeling and three-dimensional land- scape visualization system CyberCity, and image rapid roaming system 3 DBrowser. The coordinates, mass and height information of its building model are collected by aerospace images and topographic map in combination with ground digital elevation model (DEM) data, all of which contribute to accurate information concerning ground objects, and can simulate a wide range topographic and geomorphic. It can simulate large-scale landforms. The defects of this technology are that it has very limited ability to display the details of buildings and environment and weak functions in terms of light effects and texture processing.

\section{KEY TECHNOLOGIES IN THE REAL-TIME IN- TERACTIVE ROAMING OF VIRTUAL CITY}

\subsection{Three-Dimensional Visual Simulation Modeling Technology}

Three-dimensional modeling is the basic part of virtual reality. The performance of the roaming system is greatly dependent on the model quality and the modeling database's performance optimization. In accordance with different basic modeling units, there are three kinds of modeling methods:

Polygon: It uses facets to simulate surfaces so as to make three-dimensional objects of various shapes. Facets can be triangles, rectangles or other polygons [7]. This method is easy, convenient and fast, but it cannot generate smooth surfaces.

NURBS: It can establish more realistic and vivid modeling by better controlling the curve degree of object surface. This method is suitable for the creation of smooth and complicated models.

Subdivision Surface: It is used to create smooth surfaces on arbitrary meshes. Subdivision surface is defined as the limit of an infinite subdivision process, whose basic concept is subdivision. The repeated subdivision of the initial polygon mesh can generate a series of meshes that tend to converge towards the final subdivision surface.

Besides, the technologies frequently used in the modeling process also include Lathe, Extrude, Loft and BooleanOperation. As to model making, consideration should also be given to the number of model faces generated and the line arrangement at the same time when the effects are guaranteed. The smaller the number of faces is, the lighter burden it will bring for computers in the later stage and the more convenient it will be for various media to transmit such model. The reasonable and concise line arrangement of the model is one of the key factors for high quality model mapping later on.

\subsection{Texture Mapping Technology}

Texture is two-dimensional images that keep the information concerning color. Texture mapping is the process in which two-dimensional images are mapped onto threedimensional object model and then the screen drawing area of the object are formed after perspective transformation.

The texture attribute includes various parameters in relation to illumination model and surface geometry, such as surface normal vector, diffuse reflection coefficient, etc. Therefore, texture mapping technology can be performed in the following two steps: 
(1) Confirm that which parameters of the surface need to be defined to be in the texture form, namely confirming the texture attribute.

(2) Establish the mapping relation between texture space and object space and that between object space and screen space.

Transparent Texture Mapping technology integrates fusion technology and texture technology to achieve the transparent or semi-transparent effects for part of the scene in visual simulation modeling. For simulated entities of irregular shapes such as street lamps, trees and fences in the virtual scene, their texture needs to be processed with transparent texture mapping in the modeling software. Generally speaking, there are two methods of transparent texture mapping: Binboard and crossed vertical plane technology.

Seamless Splicing of Texture Mapping technology is used to process multiple pictures respectively and then splice them together to build a complete texture mapping, making sure that the joints of the spliced texture fits well in the aspect of pattern, color and light, and that the texture mapping can make smooth and reasonable transition between each two intersectant faces of the model.

\subsection{Instantiation Technology of Repeated Objects}

In complicated scenes, a large number of the same geometries greatly increase the storage space. Instantiation can solve this problem by using the processing method of matrix transformation, in which method the geometries of the same kind share the same model data and are set in different places through matrix transformation. In this way, it only needs the storage space that stores the data of a single geometry.

\subsection{Degree of Freedom (DOF) Technology}

This technology endows the model object with the ability to move. The definition of DOF is given on the basis of defining the type and range of motion. A DOF node can put all its child nodes under control to make translational or rotational motion within the set DOF range. DOF also has hierarchical structure, which lies in the layer of category nodes. When a DOF node is a child node of another DOF, such node inherits the motion and restraint of its father node at the same time. The setting of DOF mainly includes two aspects, namely the setting of local coordinate system and the setting of DOF limit.

\subsection{Level of Detail (LOD) Technology}

This technology is used to establish several models with different levels of detail for the same object. The higher the level is, the more detailedly the model shows and the more the number of polygons needed will be [8]. In real-time operation, which LOD model is displayed is determined by the distance between the viewpoint and the object [9]. LOD makes use of the feature of perspective projection, namely when the object is far away from the viewpoint, the area of its projection on the projection image plane is small. Using the model with the lowest level of detail can accelerate system processing and rendering [10]. LOD models are generally divided into two categories: static LOD models and continuous LOD models.

\subsection{Three-dimensional Scene Segmentation}

This technology is used to segment the virtual scene model into small units, and only the entities in the current model are rendered, which greatly reduces the complexity of the model, especially in large-scale and complicated scenes.

\subsection{External Reference Technology}

It is akin to directly calling the files of another existing mesh model by a pointer from database files. This technology can increase the number of similar objects without increasing operation expenses and the number of polygons. In order to facilitate the transfer of programs, external reference can adopt the relative path. For database files of large-scale scenes, if this technology is applied, they will be structurally clear and easier to be maintained and found.

\subsection{Organization and Management of Large-scale Scenes}

For large-scale scenes like virtual city, in order to be highly realistic, the geometric and texture data, spatial geometric data and terrain texture data of city building models are all in huge numbers. Speaking from the organization and management of scenes, this technology reduces the data reading time mainly by effective organization of data, $\mathrm{LOD}$ preprocessing and reasonable memory scheduling in the stage of data transfer from external storage to internal storage. Main data structures of scene data organization include Bounding Volume Hierarchy, BSP, quad-tree and Octree.

\subsection{Visibility Culling}

In large-scale scenes of virtual city, visibility culling focuses on how to rapidly cull the possibly visible or invisible elements. Although the algorithm of this kind cannot strictly decide whether elements are visible for sure, it can be used to confirm which elements are certainly invisible. According to given viewpoint and line-of-sight direction, it can easily lead to possibly visible set of scenes. In line with the factors used for visibility evaluation, there are three categories of invisible face culling, namely View-Frustum Culling, Occlusion Culling, and Back-Face Culling.

When performing the terrain roaming, the viewpoint is close to the ground and the line-of-sight direction is nearly horizontal. Most algorithms perform the occlusion calculation in such case, using technologies like horizon and incremental horizon to cull the part that makes no contribution to final imaging, which reduces the number of patches to be drawn and to increase the drawing speed.

\subsection{Particle System-based Special Effects Technology for Virtual Scene}

In virtual city scenes, the scattered fragments generated by the collision between natural phenomena like rain, snow, smog, flame or running water and irregular objects or simulations not only have complicated logical structure, but also change dynamically. Particlesystem can be used to simulate these particular fragments so as to produce favorable visual effects. It mainly uses a good deal of small particles that have certain life cycle and various attributes as basic elements to describe irregular objects. Each particle in the particle system has the following attributes: shape and size, color, transparence, life span, generation speed, initial vector, 
changes in life cycle, speed of motion, direction of motion, etc. Furthermore, many of them can vary along with the passage of time in the simulation system, and each particle will go through the process of "generation - motion - extinction". Vega special effect simulation module particle system supports the ion source in five shapes, namely Circle, square, Line, cube and sphere. Among them, the first three are all two-dimensional and lie in the XY plane, while the last two are three-dimensional.

\subsection{Path Planning of Virtual Scene Roaming}

Path planning, also called collision-free path planning, refers to mapping out a barrier-free path linking starting point and destination node according to an evaluation criterion (such as the shortest path length, the shortest travel time, the smallest energy consumption, etc) in an environment with obstructions. From the mathematical perspective, collision-free path planning can be described as the problem of calculating the extreme value of an objective function, and such objective function is the cost of the planned path and the constraint condition is to avoid colliding with obstructions. The methods include geometric method, unit decomposition method, artificial potential field method, fuzzy logic algorithm, mathematical analysis method, and other methods derived from them.

\subsection{Pickup Technology of Three-dimensional Model}

This technology is used to select certain graphic object on the screen. In the three-dimensional interactive roaming system, it often happens that a graph needs to be picked up from the screen for various operations. Scenes in the roaming system are mainly terrain and surface features. The pickup of entities is performed by improving the ray-casting algorithm. Terrain is composed of many faces, big and small, so the method of scene segmentation should be adopted when picking up objects.

\subsection{Attribute Interactive Inquiry Technology in Three- dimensional Scenes}

Spatial information inquiry means visiting the spatial entities and their spatial information described by the geographic information system in accordance with certain requirements to select from these spatial entities the ones that meet such requirements and their corresponding attributes. Three-dimensional spatial information inquiry includes the attribute-3D model inquiry and 3D model-attribute inquiry.

\subsection{Interactive Roaming Technology of Three- dimensional Scenes}

Human-computer interaction has been an important technology in the virtual city roaming system. Real-time interaction is mainly manifested in two aspects: first, users can operate entity objects in the scene and entity objects can immediately make feedback and response to users; second, when the position and angle of view of users change, the roaming engine can immediately dispatch scene database to generate and display a new scene in real time.

Walking is the most important behavior in the roaming and the main interactive method to control the change of the position of viewpoints. There're two techniques to accomplish walking: direct control directly maps the user's action in the real world through special equipments to the virtual environment such as gesture recognition technology; physical control uses physical devices to interact, such as keyboards, joysticks and steering wheels.

There are mainly two forms of roaming. The first one is free roaming, in which users can freely control the direction, visual angle and route of roaming by means of various input device like mouse and keyboard to make flexible roaming control and operation for virtual scenic spots. With full control over the roaming route and angle, users can get to any objects they're interested in without any restrictions. The second one is purposeful automatic pathfinding roaming, namely users select the starting point and destination and then roam along with the shortest path automatically generated by the system. In this process, users can similarly control the movement of the controller in four directions with the help of external device. The most difference between this and free roaming is that the forward and backward routes have been set in line with the shortest path. The essence of the shortest path problem between arbitrary two points in the scene is how to find the shortest path and then realize the rapid roaming from one point to the other without colliding with any obstructions in the scene. The most frequently used pathfinding methods in scenes are WayPoint method and NavMesh method.

\subsection{Collision Detection Technology}

Collision detection is also an integral part of the roaming system of virtual city. Such detection should be conducted in accordance with the ground and sky boundary height so as to avoid the phenomenon of plunging into underground or passing through the atmosphere in the process of interactive roaming. The model outline of buildings should also be detected so as to avoid piercing though walls. Only by timely detecting these collisions can the realistic environment be maintained. In addition, the particle system will collide with a great number of obstructions in the process, which forms the splashing phenomenon. To simulate this effect, collision detection should also be conducted between particles and obstructions. For the purpose of enhancing the efficiency and accuracy of collision detection, the hierarchical bounding box method is adopted to conduct such detection.

Collision detection is the foundation of interaction between a dynamic object and a static object or between a dynamic object and a dynamic object in virtual roaming. It involves tracking the distance between each pair of objects and if the distance is less than a certain threshold value, then the objects collide. The goal to study polyhedral collision detection algorithm is to realize a significant reduction in the number of collision detection. Roaming engines commonly use collision detection method based on the line of sight of the forward line segment detection. When the distance between the viewpoint and the object greatly exceeds the length of the line segment, simply judge that they cannot intersect, rather than seek their intersection. The detection of bounding box is a method commonly used to achieve fast collision detection. The bounding box is the minimum rectangular enclosing the virtual object with each segment parallel to the coordinate axes. It is just a simple and rough indication of the object boundary and can meet roaming with low demand for accuracy. 


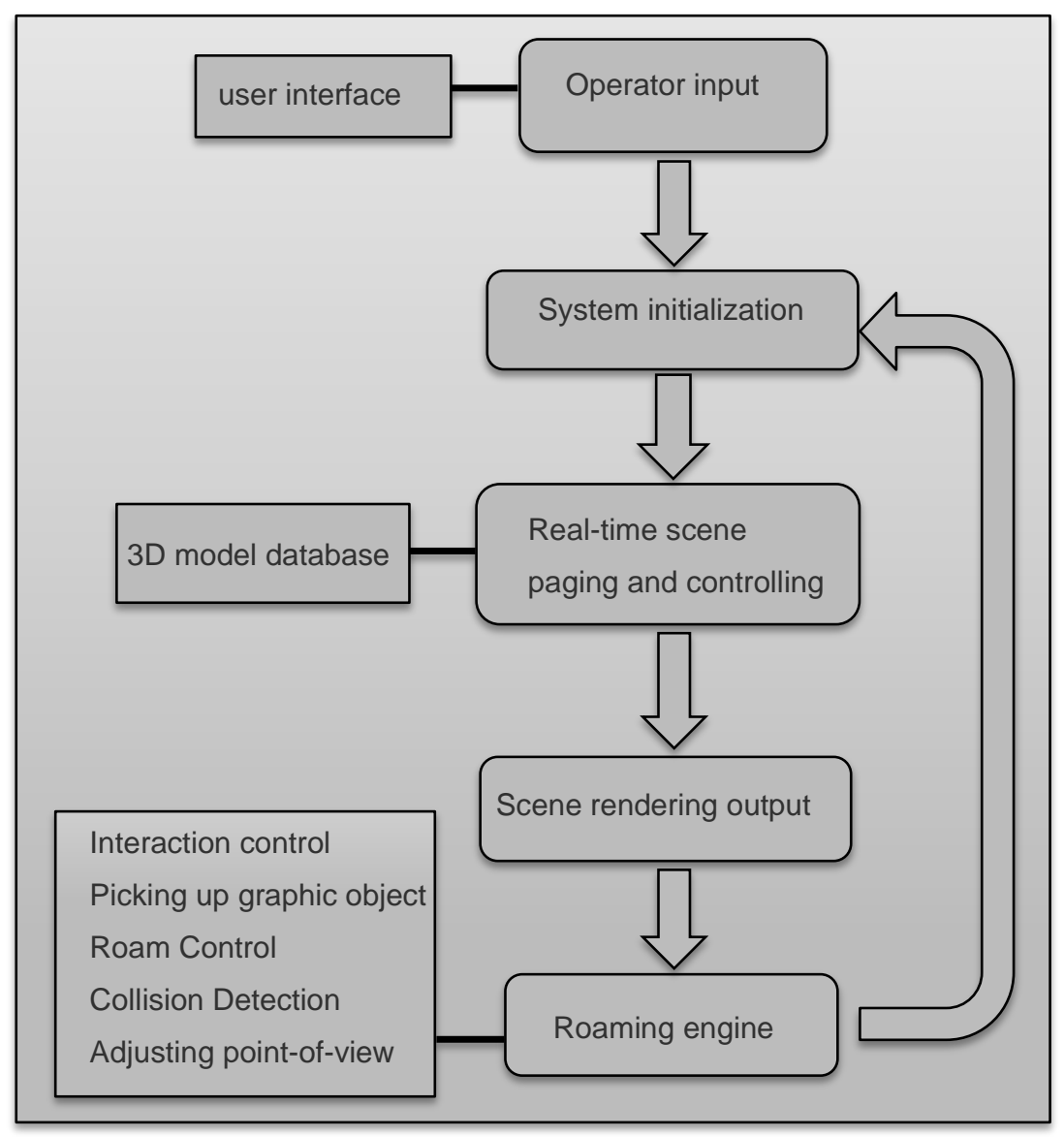

Fig. (1). Engine structure frame of the roaming system of virtual city.

\section{DESIGN OBJECTIVES AND SYSTEM STRUC- TURE FLOW}

The overall design objectives of the roaming system of virtual city are as follows:

(1) The roaming system of virtual city displays the appearance features of each building in a realistic manner, especially the major building model details [11]. It displays flowers and plants, street lamps, trash cans, bulletin board, enclosure, and etc by using various methods, and it also adds special scenes like weather effects. The constituted virtual scene includes terrain, buildings of all kinds, flowers, plants and trees, natural phenomena and so on, which enable users to feel like being personally in the virtual environment with strong sense of immersion.

(2) The roaming system of virtual city should be realistic to some extent and it should use accurate building data that has real coordinates [12]. Such data should be the data with certain accuracy obtained by various surveying and mapping methods. Scenes of virtual city should be basically consistent with real city scenes, and buildings should be built in the computer in accordance with actual dimension in the real world.

(3) On that basis, mathematical model and engine development should be used to complete the roaming design, ena- bling users to roam in the virtual scene in different ways that live up to the needs of users and to offer convenient mouse and keyboard operations. In this way, users can roam along with the preset fixed path in the three-dimensional scene and perform interactive roaming for the buildings they are interested in as well. In the roaming process, multiple ways of motion can be selected, such as walking, driving, flying, etc. Besides, users can also perceive the changes in natural environment such as sky in such process. Fig. (1) shows the engine structure frame of virtual city roaming.

\section{THE PROCEDURE TO IMPLEMENT THE ROAM- ING SYSTEM OF VIRTUAL CITY}

The design of the roaming system of virtual city mainly takes into account two aspects, namely the three-dimensional scene modeling and the establishment of the roaming system. The process can be roughly divided into the following steps: (Fig. 2)

(1) Firstly make clear of application type, model size, software to be used, hardware to be equipped, and etc.

(2) Collect and preprocess the original data. Obtain a huge number of model data information, including scene feature data, terrain data, feature data, building layout and texture images, and then preprocess these original data to cull the data with large deviations $[4,13]$. 


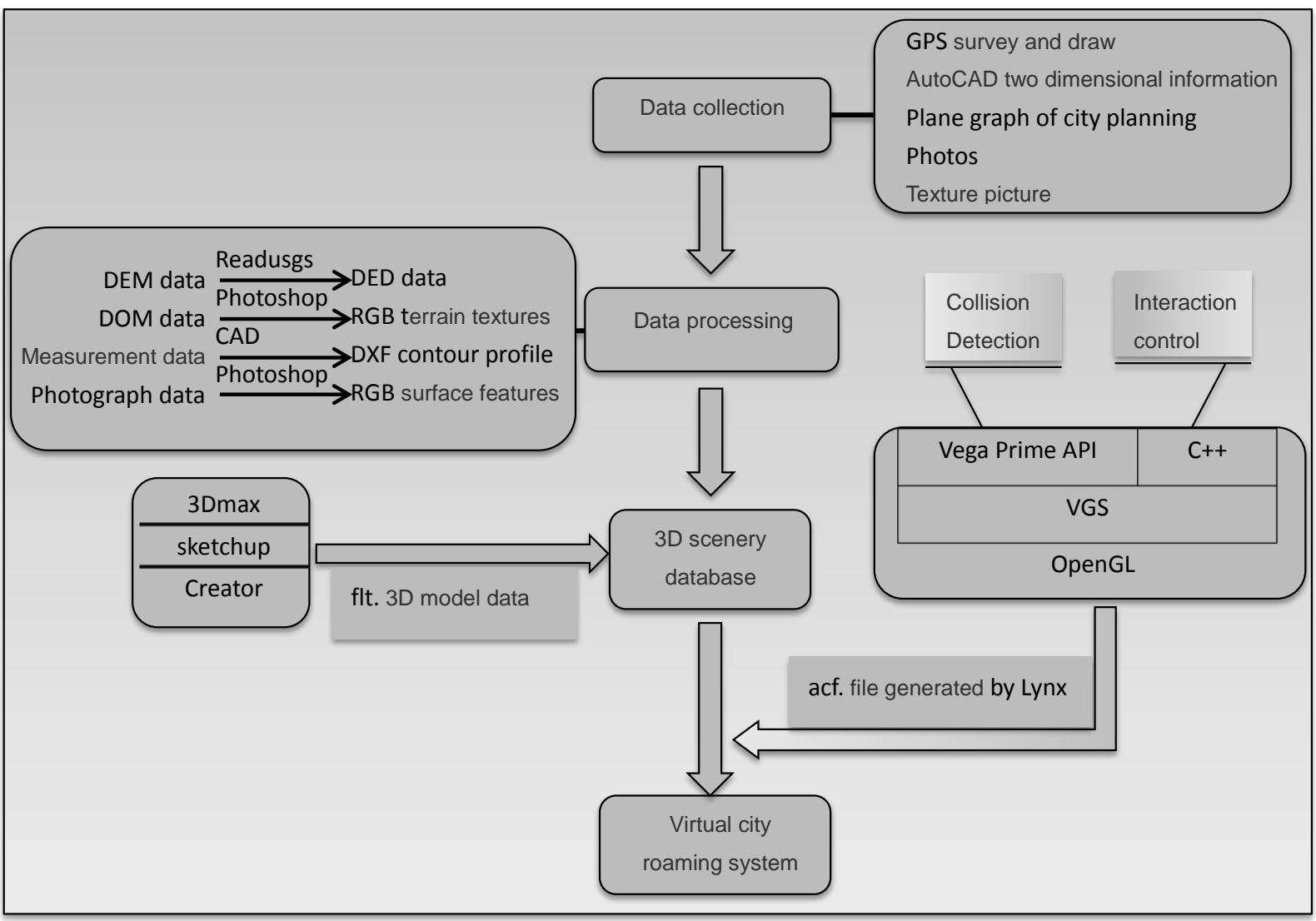

Fig. (2). The procedure of the roaming system of virtual city.

(3) Create model database and conduct optimization. Select the development platform, carry out the threedimensional modeling by using modeling tools such as Creator, GoogleSketchUp [14], CAD, 3dsMax and Maya, and save the created scene models that include terrain surface model, single object model, building model and plant model in .flt format, so as to form attributive characters of the scene's entity model. After the above steps are performed, the virtual scene thus created should be integrated and the model optimized [15].

(4) Realize the interactive roaming. Add various virtual scenes, virtual observers and way of motion in Vega, write the driver program in Vega codes, generate the application programs in .adf format, set the method of collision detection, arrange the path roaming way, and finally generate executable program by using $\mathrm{VC}$ software, thus realizing the roaming in the entire virtual city.

(5) Output to release. Select the type of release and output it to put into practical use.

\section{HOW TO MAKE USE OF VEGA TO ACHIEVE VIRTUAL CITY ROAMING}

\subsection{The introduction of the Scene Development Envi- ronment of Vega}

A lot of high-level three-dimensional scene development environment and software supporting real-time three- dimensional processing appears based on the underlying OpenGL, among which, currently, three more often used software is SGI Company's IRIS Performer, CGZ Company's VTree and MultiGen-Paradigm's Vega. Vega software has a friendly graphical environment interface, complete $\mathrm{C} / \mathrm{C}++$ language application programming interface API, rich library functions and a large number of functional modules. It combines advanced simulation, special analog functions and easy-to-use tools to make users use simple operations to create, edit and run complex simulation programs and provides a convenient real-time means of processing complex simulation events. Vega largely reduces source code and its development time, has good visual programming environment, and make it easy to use for programmers and non-programmers. Engineers, designers, planners and developers can use the real-time simulation technology to combine with their design.

\subsection{Vega Software's Components and Working Principle}

Vega mainly consists of two parts: the toolbox of the LynX graphical user interface and the library of functions based on the $\mathrm{C}$ language. Vega contains basic modules and optional modules for different simulation applications. Object-oriented technology defines a variety of graphics management features and optional modules as "class". Vega provides common functions for various public uses and simplifies the programming development. The click graphic environment of Lynx can quickly and easily change the applica- 
tion performance, video channel, multi-CPU allocation, viewpoints, viewer, special effect, different time, system configuration, model and database without writing source code. Lynx can be extended into user-defined panel and functions to meet the special requirements of users, to reconfigure the system and to provide a more economical solution. The finally generated ADF contains three types of data: keywords are the set of control parameters of attributes in a class; parameters are values of a particular item in keywords; a class is a set of related keywords. ADF file is a full description of the graphical environment and database components composing visual simulation applications and can be run directly and generate simple simulation applications [16].

The working principle based on Vega development realtime applications is as follows: use ADF to undertake initialization settings, edit the simulation program, and dispatch functions of the initialization system and module; read into data of ADF and load the relevant database, create and configure instances of a class and dispatch frame loop function to perform rendering of relevant functions' program library; refresh the scene. Once various instances are created, they will be added to the list of items and all instances can be searched by the name of the index; so when users get the instance's handle, they can process its properties.

\subsection{Data development procedure of virtual city roaming system based on Vega}

The implementation of roaming system can be generally simplified into two steps: first, complete 3D scene modeling and then construct roaming engine to complete the visual output and roaming control of the scene. To use Vega application to develop three-dimensional real-time simulation applications includes the following process: first, determine its scope, geographic location, emulation type, effect requirements and implementation platform; collect source data, terrain data, feature data, models and image data; according to the different requirements, choose different resolution and accuracy. Then create real-time three-dimensional database which consists of a single model and a terrain surface model; use Creator create and determine the level detail structure of the model and store it as the format of OpenFIight file. Finally, finish the real-time application development; dispatch the three-dimensional database into Vega, define the display and movement patterns of objects, increase the environmental effects, establish the application definition file ADF, and on the basis of ADF, develop functions required by users.

The four types of involved raw data are digital elevation model (DEM), digital orthophoto map (DOM), surface features measurement geometry data and surface features photos. The specific workflow and technology route of conversion process is as follows: express the natural terrain by using the integration of DEM and DOM; use Readjusts tool of Creator to convert to be DED data and then carry out the conversion of the terrain model. Convert terrain DOM with Photoshop to be RGB file and associate it with terrain in Creator to get the textured terrain. Use CAD software to record measurement data to be digital line graph in DXF format and then import to the Creator to undertake modeling for surface features based on it. The photographs also need to use Photoshop to convert into the required RGB texture file and associate it to the corresponding feature. Finally, realize special effects with Vega programming and modify and improve the model.

\subsection{Specific steps to achieve Vega-based virtual city roaming system}

On the basis of three-dimensional modeling of virtual cities, using Vega to undertake secondary development can build a dedicated system to more vividly reproduce the style of the city and building features. Vega provides two ways to achieve the real-time drive in virtual scene: one is visual programming and the other is Vega API functional programming. Concrete steps of the visual programming approach are as follows: select settings tool in the toolbox of the Lynx graphical interface and undertake the corresponding parameter settings. Select objects and scenes and set the terrain, buildings and all objects to be displayed in the virtual scene. Then select the observer and movement mode and then set the way of observing and movement mode of the observer in the virtual scene. Set the environmental effects, moving targets, light source, sound effects, collision detection and others. Finally, run these settings and a mouse can be used to control the advancing direction and roam in the virtual scene. It provides different ways to place observers: Tether-follow, Tether-Spin, Tether-Fixed, Path Navigator, Motion Model and Manual which allow an observer to move following the model or along the trajectory path, or in a fixed position and direction.

\section{CONCLUSIONS}

As a visual manifestation of digital cities, virtual city roaming system plays a huge role in the fields of urban planning and management, reporting, approval, design and image promotion. The technology enables governmental planning departments, project developers, engineers and the public to review planning effects from any point of view with realtime interaction, to have a better grasp of the urban form and to understand the design intent of planners. Existing programs can be exported as video files to be used to create multimedia information to be publicized. When users roam and interact in the 3D scene, many subtle design flaws difficult to detect can easily be found to reduce the losses caused due to the incompleteness of the previous planning and to improve the quality of the assessment of a project. To modify parameters can easily re-design and amend a program to improve efficiency and save money. The virtual city roaming system enables complex systems of urban geography, resources, environment, ecology, population, economy and society to achieve visualization and virtualization and to be network-based, so that urban planning has higher efficiency, richer performance practices and more information; besides, it can improve the efficiency of the urban construction and the effectiveness of urban management and promote the sustainable development of cities.

\section{CONFLICT OF INTEREST}

The authors confirm that this article content has no conflicts of interest.

\section{ACKNOWLEDGEMENTS}

Declared none. 


\section{REFERENCES}

[1] K. Oh, "Landscape information system: A GIS approach to managing urban development", Landscape and Urban Planning, vol. 54, no. 1-4, pp. 79-89, 2001.

[2] J. Rossignac and P. Borrel, "Multi-resolution 3D approximations for rendering complex scenes", Springer, US, 1993.

[3] T. Barbieri and P. Paolini "Reconstructing Leonardo's Ideal City From Handwritten Codexes to Webtalk-II: A 3D Collaborative Virtual Environment System", In: Proceedings VAST 2001 Virtual Reality, Archeology, and Cultural Heritage, pp. 61-66, 2001.

[4] J. Hu, S. You and U. Neumann, "Approaches to Large-Scale Urban Modeling", IEEE Computer Graphics and Applications, vol. 23, no. 6, pp 62-69, 2003.

[5] K. Ikeuchi, M. Sakauchi, H. Kawasaki, and I. Sato, "Constructing virtual cities by using panoramic images", International Journal of Computer Vision, vol. 58, no 3, pp. 237-247, 2004.

[6] W. Correa, J. Klosowski and C. Silva: "Inieractive Out-of-Core Rendering of Large Models", Technical Report", TR-653-02, Princeton University, 2002.

[7] P. Liu and Z. Peng, "Three-dimensional modeling of virtual city: Taking multiGenCreator for example", Advanced Materials Research, vol. 711, pp. 617-622, 2013.

[8] V. Coors, "3D-GIS in networking environments", Computers, Environment and Urban Systems, vol. 27, no. 4, pp. 345-357, 2003.
[9] D. Luebke, "Level of Detail for 3D Graphics", Morgan Kaufmann Publishers. M.A, USA, 2003.

[10] J. C. Xia, J. El-Sana, and A. Varshney, "Adaptive real-time levelof-detail-based rendering for polygonal models", IEEE Transactions on Visualization and Computer Graphics, vol. 3, no. 2, pp. 171-183, 1997.

[11] K. Lee, "3D urban modeling and rendering with high resolution remote sensing imagery on mobile $3 \mathrm{D}$ and web $3 \mathrm{D}$ environments system architecture and prototype implementation", In: $2007 \mathrm{Ur}$ ban Remote Sensing Joint Event, URS, 2007.

[12] S. Singhal and M. Zyda, "Networked Virtual Environments Design and Implementation", Addison Wesley, Reading, MA, USA 1999.

[13] V. Coors, "3D-GIS in networking environments" Computers, Environment and Urban Systems", vol. 27, no. 4, pp. 345-357, 2003.

[14] J. Binder, "Earth to Google: Satellite images on your screen" Aerospace America, vol. 43, no. 11, pp. 18-20, 2005.

[15] C. He, G. Jianguo and P. Ping, "Experiment and research of google SketchUp combine with ArcGIS in the Three-Dimensional urban geographic information system", In: WCSE'09. WRI World Congress, 2009.

[16] P. Liu and Z. Peng, "The realization of virtual city roaming based on vage", Applied Mechanics and Materials, vol. 321-324, pp. 1971-1976, 2013.

Received: August 13, 2013

Revised: October 02, 2013

Accepted: October 02, 2013

(C) Liu and Peng; Licensee Bentham Open.

This is an open access article licensed under the terms of the Creative Commons Attribution Non-Commercial License (http://creativecommons.org/licenses/by-nc/3.0/) which permits unrestricted, non-commercial use, distribution and reproduction in any medium, provided the work is properly cited. 